\title{
Implementation of electronic stethoscope for online remote monitoring with mobile application
}

\author{
Sugondo Hadiyoso, Dieny Rofiatul Mardiyah, Dadan Nur Ramadan, Asril Ibrahim
}

School of Applied Science, Telkom University, Indonesia

\begin{tabular}{l} 
Article Info \\
\hline Article history: \\
Received Oct 27, 2019 \\
Revised Feb 13, 2020 \\
Accepted Mar 23, 2020
\end{tabular}

\section{Keywords:}

Android

Auscultation

Cloud server

Electronic stethoscope

Online

\begin{abstract}
The stethoscope is a piece of medical standard equipment that isused by a physician for an initial examination of the patient. Generally, the stethoscope is used for auscultating sounds which are generated by the workings of organ systems such as cardiac, lung or digestive. In the present condition with the growing number of the patient population, it has an impact on the burden of hospitals and medical practitioners. So that treatment is not optimal, especially patients who need continuous monitoring. Thus it needs a system that can work dynamically, flexibly and remotely based. This paper focuses on the implementation of the electronic stethoscope which is integrated with a mobile phone as the modality of online data transmission through the internet network. The prototype of an electronic stethoscope uses condenser mic, pre-amplifier, wide bandpass filter $(20 \mathrm{~Hz}-1 \mathrm{KHz})$ and audio amplifier. The maximum gain is $28.63 \mathrm{~dB}$ in the $20 \mathrm{~Hz}-690 \mathrm{~Hz}$ frequency range. The signal output can be connected to the android mobile through the jacked phone to be stored in MP3 format and then sent to the cloud server for further monitoring and analysis. The application called "Steder" supports realtime communication between patient and physician for medical check-up, consultation, and discussion activities.
\end{abstract}

This is an open access article under the CC BY-SA license.

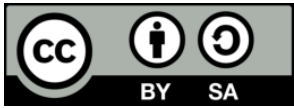

\section{Corresponding Author:}

Sugondo Hadiyoso,

School of Applied Science,

Telkom University,

Telekomunikasi Road, Bandung, West Java, Indonesia.

Email: sugondo@telkomuniversity.ac.id

\section{INTRODUCTION}

The stethoscope is the main device of medical practitioners [1], commonly used to determine the initial condition of the patient's health. Object observations are usually the sound of heart, lung and digestive organs. This technique is usually called auscultation [2]. To improve the quality and demands of data recording, an electronic stethoscope device was developed. An electronic stethoscope with a loudspeaker allows the sound to be heard clearly in real-time [2-5]. Electronic stethoscopes are becoming a new challenge in the medical device industry that can improve the efficiency and capability of captured, stored and played back [2]. With the existence of electronic stethoscope technology, someone can check their own health condition then consult with medical experts by sending sound recordings.

Recently, people's awareness about the importance of health is more considerable by conducting regular checks and consultations to the physician. However, some others with their occupations sometimes do not pay attention to the health condition of their own body. Some people feel sick, but they can not afford to go out, the solution is to take a rest at home or try to treat themselves. Surely if this does not get the right 
action can make the condition worse. To solve the problem, an integrated system is needed to facilitate patients and medical practitioners to monitor via desktop PCs as in research [1, 6-9].

On the other side, the advancement of the internet of things (IoT) technology and applications that support smartphones has become one of the main issues. One of them is android smartphones that reach a hundred million users. Smart applications on mobile phones have provided many benefits and make it easy for users. Devices or objects in the world to be connected through the internet based on IoT Technology [10]. Applications in the medical field can be called the internet of health things (IoHT) [11, 12] or Internet of medical things (IoMT) [13]. In the present real situation, the population continues to grow and the related rise in chronic illness affects the burden on hospitals, doctors, and nurses [14, 15]. This can also have an impact on the patient's limited interaction with the doctor and the difficulty of monitoring the patient's health continuously, especially in patients with serious illnesses.

Some research has proposed a monitoring system of the body's vital parameters (for example heart rate, respiratory rate, oxygen saturation, blood pressure or body temperature) based on IoT [16-23]. However, less research has proposed an IoT-based auscultation system. Related research to electronic stethoscopes with cloud server systems has been proposed in [24, 25], which is PC-based data processing. This system certainly has low mobility because it depends on the PC. Therefore in this paper, we propose an integrated auscultation system that has high mobility. This is considered to be very necessary due to the lack of resources related to the auscultation system in the IoT application. An electronic stethoscope that is integrated with mobile Android can be used as an alternative for medical monitoring applications. This study discussed the design of electronic stethoscope hardware and android applications that can record stethoscope sound, convert to mp3 format and send the sound file to the cloud server to be accessed online through the internet network.

The design of the electronic stethoscope in this research consists of a condenser mic mounted on an acoustic stethoscope tube, pre-amplifier, wide bandpass filter $(20 \mathrm{~Hz}-1 \mathrm{KHz})$ and an audio amplifier. The electronic stethoscope is connected to the mobile device through the jacked phone then through the application, the sound of the examination results can be recorded and converted to MP3 format to be sent to the internet cloud. Patients will send the data to the doctor so that doctors can provide the diagnosis in real-time using the mobile application. This system is expected to support the application of telehealth monitoring and awareness of a person to health to improve the quality and health services.

The rest of the paper is organized as follows. Section 2 describes the proposed system which was designed and realized in this study. Section 3 explains the result and discussion of this study. Finally, section 4 is the conclusion of this study.

\section{PROPOSED SYSTEM}

The proposed system consists of an electronic stethoscope and an application called "Steder" (stethoscope reader) on the Android mobile phone. The electronic stethoscope takes sound and converts to electrical signals to be processed, stored and sent to the cloud by Steder. Steder application runs on an Android-based smartphone using minimum version Ice Cream Sandwich. The application is integrated with Google Firebase as the cloud database server. Where all patient data will be stored in the Firebase Real-Time Database, meanwhile the results of sound recordings will be stored in the Cloud Storage feature in Firebase. This system involves patients and doctors or nurses. Of course, they are responsible for the availability of this service from initiation to the application running. Ownership of electronic stethoscope hardware will be charged to the patient. Patients or their families have previously been given simple knowledge and training on the use of a stethoscope and auscultation techniques so that they can carry out their examinations. Guidelines in the form of text and images are of course also provided. The patient will record the sound of heart, respiration or other body regions and finally complete the form as a requirement to consult with a doctor. If the patient has finished recording and filling out the form of consultation, then the patient will send the data to the doctor. Doctors will retrieve or download recordings from cloud storage. Then the doctor will read the patient's symptoms and analyze the recorded sound. Finally, the doctor will send the diagnosis to the patient. The illustration of the proposed system can be seen in Figure 1.

\subsection{Electronicstethoscope design}

Electronic stethoscopes are the main devices in this proposed system. The stethoscope is designed for the acquisition of heart sound, respiratory and other body regions in the range of $20-1000 \mathrm{~Hz}$. The maximum output current is $1.3 \mathrm{~mA}$, this is adjusted to the audio phone's current input. The components used for the design of electronic Stethoscope are membrane, condenser mic, pre-amplifier (gain $26 \mathrm{~dB}$ ), wide bandpass filter $(20-1000 \mathrm{~Hz})$, audio power amplifier (gain $2 \mathrm{~dB}$ ) and jack phone. 

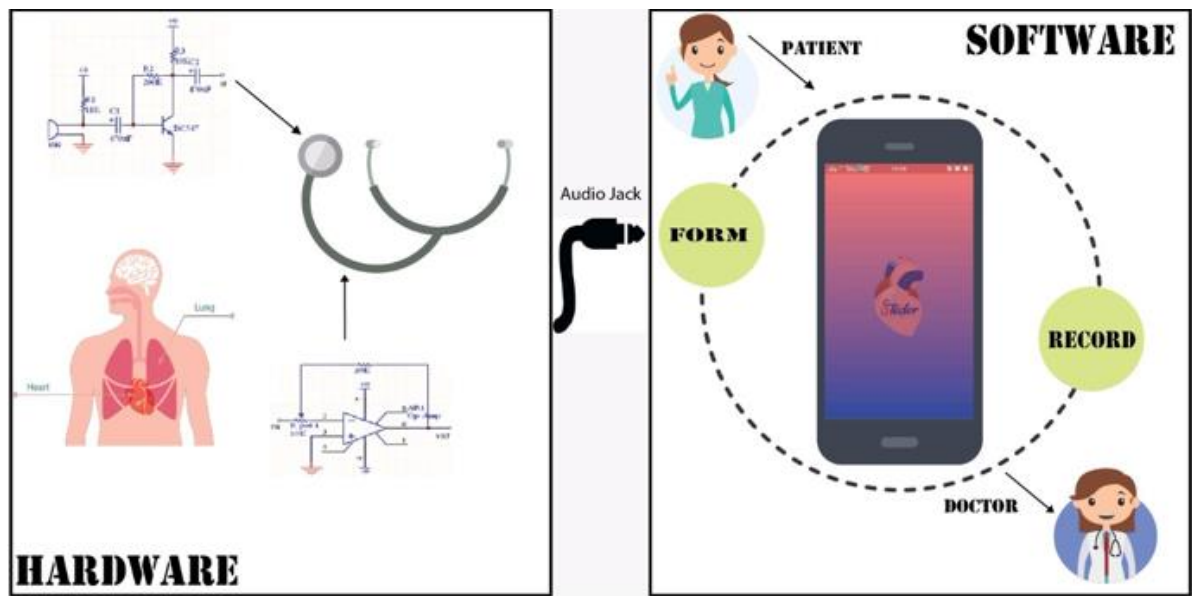

Figure 1. Proposed system

\subsection{Stethoscopereader (steder) application}

Steder is an Android-based mobile application. This application has a feature that can record sound from an electronic stethoscope. Through this application, sound recordings can be stored, downloaded and played back so that doctors can analyze the patient's health condition. The output of this application is a data file, whether it is patient data or sound record in MP3 format. The flow process of this application is described in Figure 2. The flow process of this application is as follows.

- $\quad$ Users must register before login and input data such as name, date of birth, e-mail and password.

- $\quad$ Login according to user authorization whether patient or doctor. The doctor's data will be verified first by the admin before the doctor can $\log$ in as a user.

- $\quad$ After login, patients and doctors can send messages to each other, make complaints on the patient's side and the doctor can send a diagnosis.

- $\quad$ Patients are required to choose a doctor according to the list.

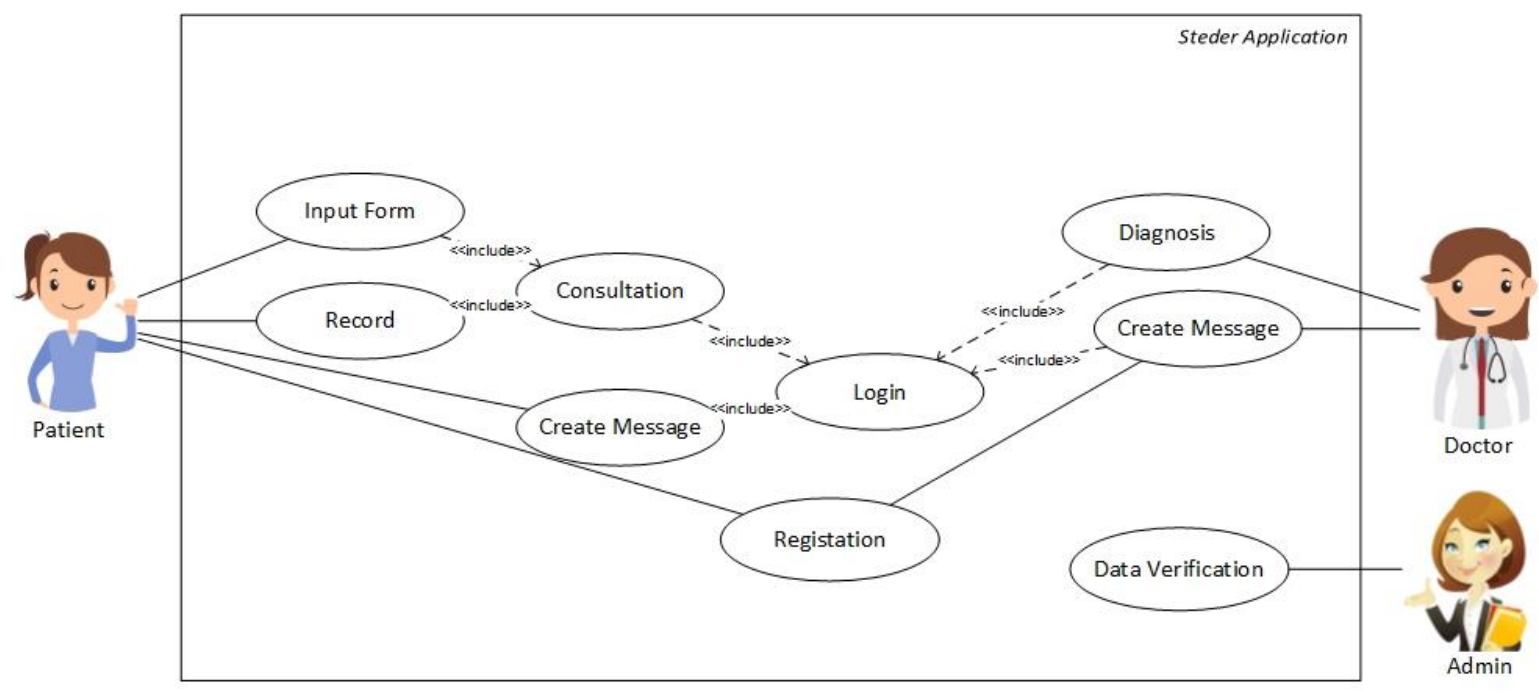

Figure 2. Use case diagram

The design of the site map application on Steder shown in Figure 3. It can be seen that in the application several menus are related to each other. Where the menus are grouped by user authorization. As is known user here is grouped into two groups of patients and doctors. 


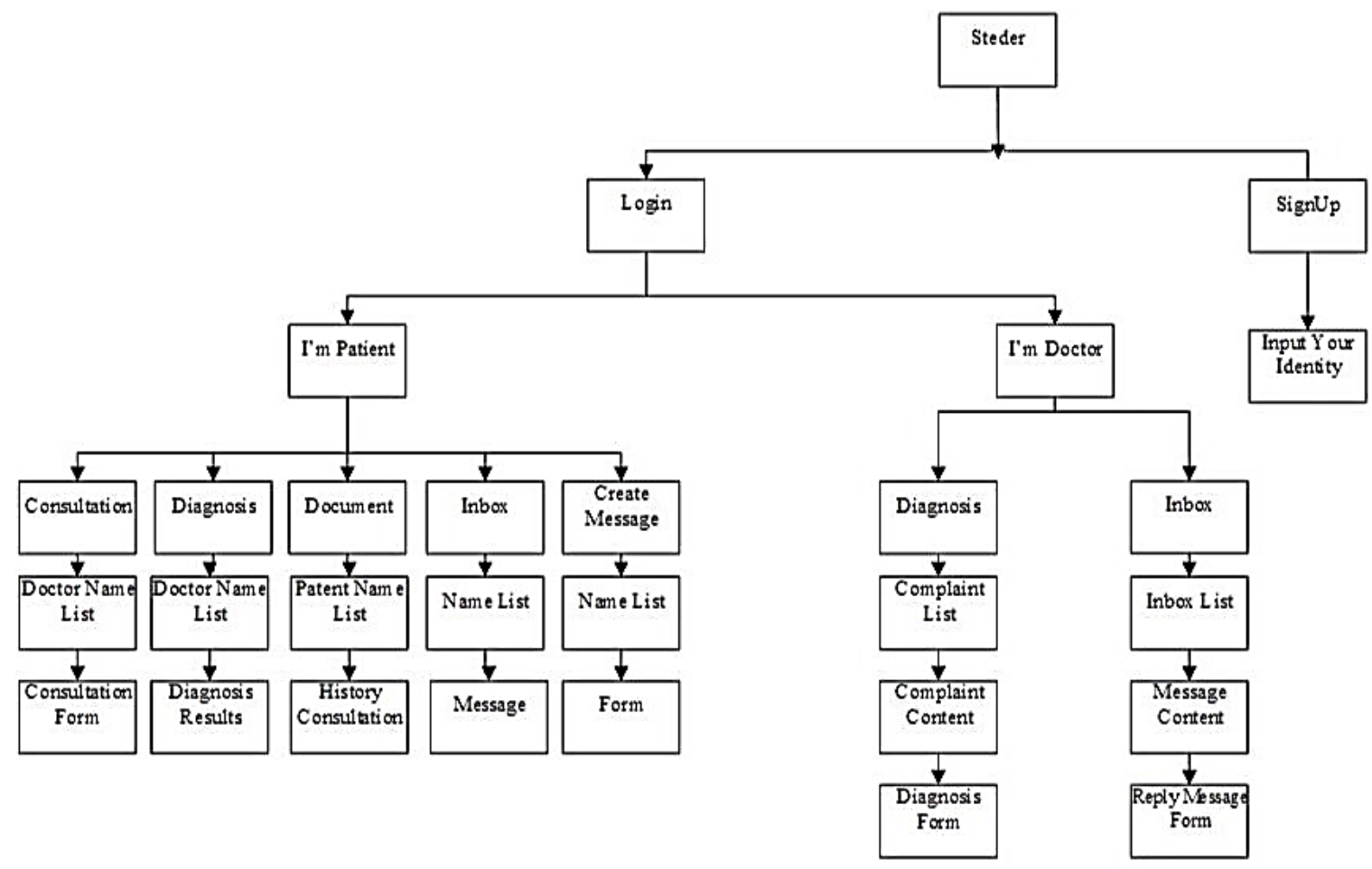

Figure 3. Site map application

\subsection{Testing scenarios}

The performance test on this proposed system is hardware and application testing. Electronic stethoscope hardware testing includes gain, filter response, and signal form. Tests performed on the application are minimum operating system requirements, voice recording, functionality, integration testing on firebase, download and upload testing, delay, and conformity of audio files. Based on Figure 4, a test perform is conducted involving two sides of the user ie patient and doctor. The parameters tested are all functionalities both hardware and applications, delay and integration with Firebase.

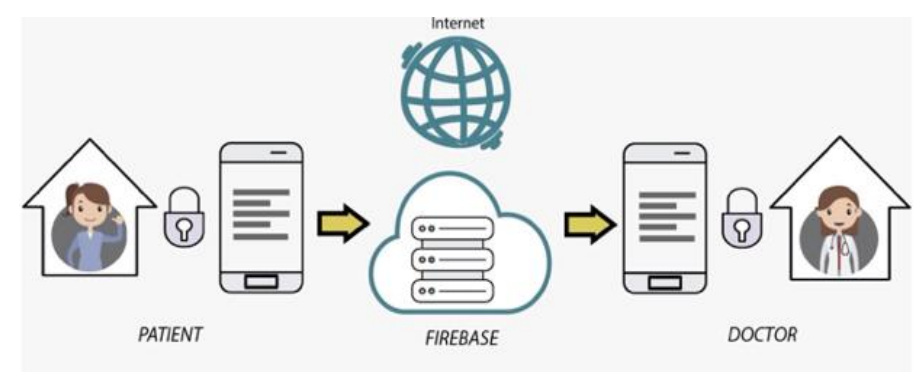

Figure 4. Performance test

\section{RESULTS AND DISCUSSION}

\subsection{Hardwaretesting}

Testing and analysis of an electronic stethoscope are conducted to determine the performance capability of the device that has been realized. Measurement of each part of this electronic stethoscope uses DC power supply, function generator, and oscilloscope. Hardware testing includes filter response, amplifier and auscultation test. Filter measurements aim to find out the cutoff frequency and frequency response generated by HPF and LPF functionalized into BPF filters. Measurement of filter response was done by using the function generator and oscilloscope. The input signal voltage is kept constant at $1.05 \mathrm{Vpp}$ while the output frequency is measured. The cut-off frequency is determined when the output voltage is $0.707 \mathrm{Vpp}$. The BPF frequency response is shown in Figure 5. 
Figure 5 shows that the realized BPF has a lower cut-off frequency of $20 \mathrm{~Hz}$ and an upper cut-off of $960 \mathrm{~Hz}$. For lower cut-off frequency results are obtained according to design and upper cut-off frequency lower than $\approx 40 \mathrm{~Hz}$ when compared to designs with a bandwidth of $20-1000 \mathrm{~Hz}$ but these frequency bands still meet to pass the heart and breathing sounds. In the measurement of the audio amplifier circuit obtained the following results:

- $\quad$ Input Level : $3 \mathrm{Vpp}$

- Output Level : $6 \mathrm{Vpp}$

So that the gain of the audio amplifier is 2 times or $3.01 \mathrm{~dB}$. This output voltage is sufficient as an input analog audio signal on the smartphone. Then the overall performance of electronic stethoscopes was tested. This measurement can be known as the sample signal output sound of heart and breathing through an oscilloscope. The results of the measurement can be shown in Figure 6 . The results of the measurement can be shown in Figure 6.

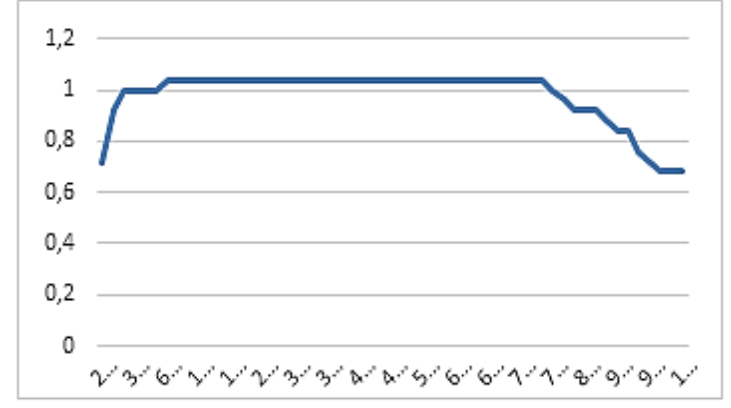

Figure 5. The frequency response of BPF

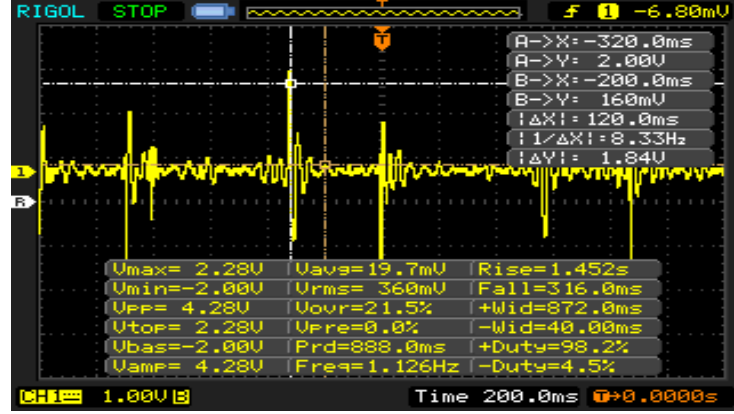

Figure 6. Heart sound signal on the oscilloscope

\subsection{Application testing}

\subsubsection{Minimum version of android}

The designed Steder app will run optimally on the minimum version of the ice cream sandwich (Ver. 4.0). Testing is done using 5 smartphones that have different android versions with the purpose to know the success of the installation. Test results can be shown in Table 1. Steder application data size of apk format that is equal to $4.05 \mathrm{MB}$. While after the installation process, the memory capacity used by this application is 19.9 MB. Application steder requires 42MB of RAM space when running. From the test results shown in Table 1, the smartphone that has the operating system version under ice cream sandwich that is honeycomb, can not install steder app. Based on the results of the test in Table 1, the application made is in accordance with the initial design.

Table 1 . Android minimum version test result

\begin{tabular}{cc}
\hline Version & Installation \\
\hline Honeycomb & Not installed \\
Ice Cream Sandwich & installed \\
Jellybean & installed \\
Kitkat & installed \\
Lollipop & installed \\
Marshmallow & installed \\
Nougat & installed \\
\hline
\end{tabular}

\subsubsection{Application integration with database}

Tests conducted to find out the features on the application can run well and following the application interface and database that has been created.

- Application integration with authentication

This authentication process is useful for the user login process to application. The authentication process is done on the database to match the user ID and password so that the user can access it according to their authorization. In the database, the user account is encrypted to strengthen system security as seen in Figure 7.

- Application integration with real-time database 
This test was done to ensure that the data sent is data stored in the Firebase Real-Time Database. It aims to simplify the system in the retrieval and storage of data in real time. The data successfully stored in the database can be seen in Figure 8.

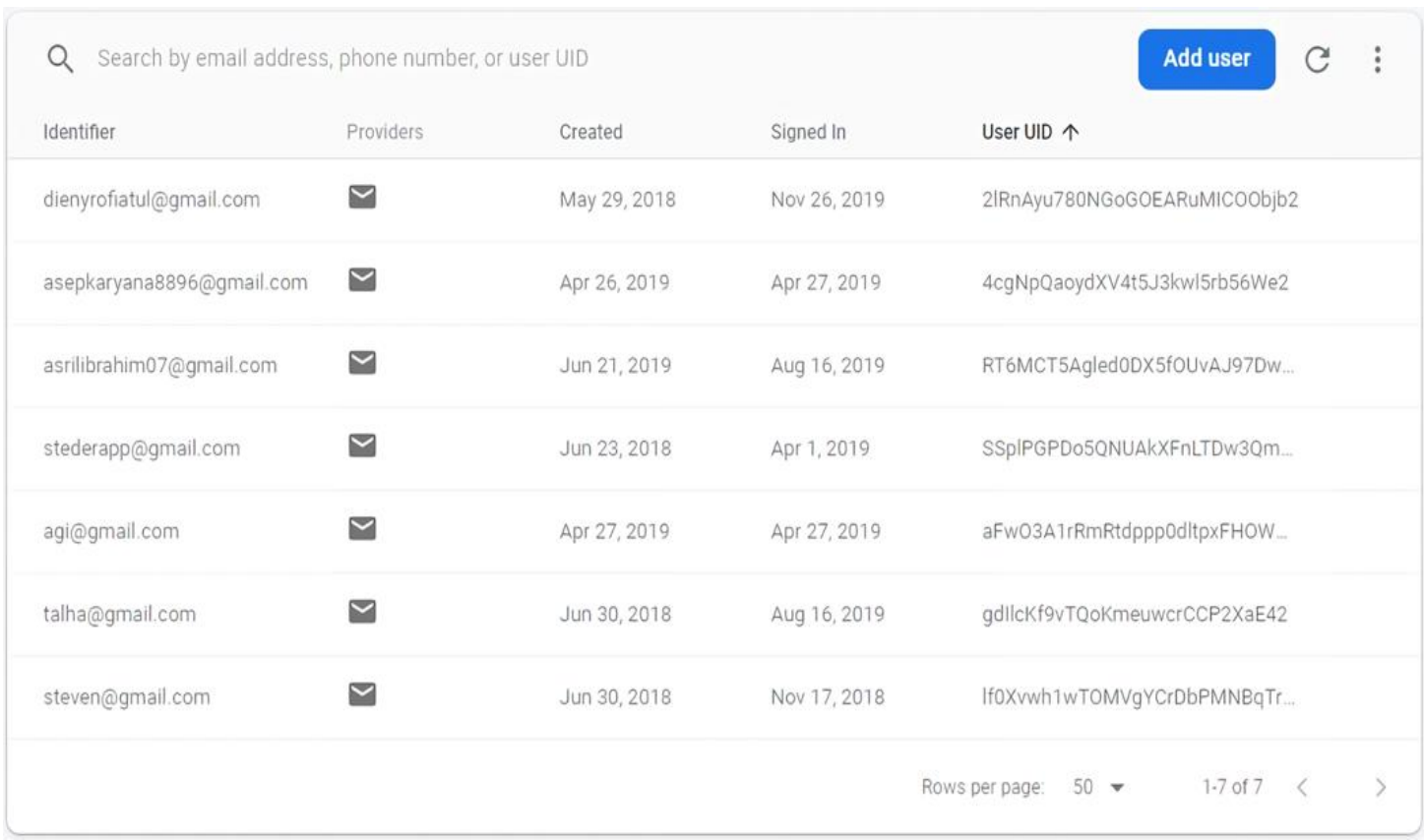

Figure 7. Firebase authentication

\begin{tabular}{|c|c|c|c|c|}
\hline & gs://stederapp-97bgf.appspot.com > Audio_File & & & 스 Upload file \\
\hline$\square$ & Name & Size & Type & Last modified \\
\hline$\square$ & ๑ 06624 AudioRecording.mp3 & $156.73 \mathrm{~KB}$ & audio/mp3 & Nov 17,2018 \\
\hline$\square$ & 07411AudioRecording.mp3 & $16.69 \mathrm{~KB}$ & video/3gpp & Jul 31, 2019 \\
\hline$\square$ & 15379AudioRecording.mp3 & $92.36 \mathrm{~KB}$ & video/3gpp & Aug 1, 2019 \\
\hline$\square$ & 28281AudioRecording.mp3 & $6.58 \mathrm{~KB}$ & video/3gpp & Apr 26, 2019 \\
\hline$\square$ & ๑ 51160AudioRecording.mp3 & $151.84 \mathrm{~KB}$ & audio/mp3 & Nov 17,2018 \\
\hline$\square$ & D3540AudioRecording.mp3 & $13.63 \mathrm{~KB}$ & video/3gpp & Apr 27,2019 \\
\hline$\square$ & 60641AudioRecording.mp3 & $21.07 \mathrm{~KB}$ & video/3gpp & Apr 27,2019 \\
\hline$\square$ & 90758AudioRecording.mp3 & $13.82 \mathrm{~KB}$ & video/3gpp & Apr 26, 2019 \\
\hline
\end{tabular}

Figure 8. Stored data

\subsubsection{Audio file size testing}

This test is done to determine the size of the audio file uploaded to the database. The test was performed by recording stethoscope sounds for 60 seconds at 20 experiments. The results of the test can be seen in Table 2.From Table 2 above it is concluded, the average file size of sound recording in 60 seconds is 734,187 bytes. 
Table 2. Sound recorder size

\begin{tabular}{|c|c|c|c|c|c|}
\hline No & File Name & Size & No & File Name & Size \\
\hline 1 & 01636AudioRecording.mp3 & 733,997 bytes & 11 & 33310AudioRecording.mp3 & 734,206 bytes \\
\hline 2 & 02817AudioRecording.mp3 & 734,187 bytes & 12 & 40317AudioRecording.mp3 & 734,231 bytes \\
\hline 3 & 04742AudioRecording.mp3 & 734,298 bytes & 13 & 41331 AudioRecording.mp3 & 734,135 bytes \\
\hline 4 & 18829AudioRecording.mp3 & 734,341 bytes & 14 & 59144AudioRecording.mp3 & 733,762 bytes \\
\hline 5 & 22508AudioRecording.mp3 & 734,184 bytes & 15 & 66410AudioRecording.mp3 & 734,161 bytes \\
\hline 6 & 24824AudioRecording.mp3 & 734,207 bytes & 16 & 71677AudioRecording.mp3 & 734,296 bytes \\
\hline 7 & 25549AudioRecording.mp3 & 734,235 bytes & 17 & 73085AudioRecording.mp3 & 734,223 bytes \\
\hline 8 & 27184AudioRecording.mp3 & 734,180 bytes & 18 & 76534AudioRecording.mp3 & 734,305 bytes \\
\hline 9 & 31866AudioRecording.mp3 & 734,147 bytes & 19 & 92075AudioRecording.mp3 & 734,241 bytes \\
\hline 10 & 33211AudioRecording.mp3 & 734,227 bytes & 20 & 99278AudioRecording.mp3 & 734,184 bytes \\
\hline
\end{tabular}

\subsubsection{Download file testing}

Audio download test aims to know that audio files that are in cloud storage successfully downloaded by a doctor. The results of this scenario test can be seen in Table 3. From the test results shown in Table 3, it can be concluded that the audio playback on the side of the doctor in accordance with system design. So the audio played following the patient file being observed.

Table 3. Audio file download

\begin{tabular}{cccc}
\hline No & File Name & Download File Status & File Playback Compatibility \\
\hline 1. & 15843AudioRecording.mp3 & Success & Match \\
2. & 28235AudioRecording.mp3 & Success & Match \\
3. & 40268AudioRecording.mp3 & Success & Match \\
4. & 43758AudioRecording.mp3 & Success & Match \\
5. & 55719AudioRecording.mp3 & Success & Match \\
6. & 75800AudioRecording.mp3 & Success & Match \\
\hline
\end{tabular}

\subsubsection{Diagnostic menu}

The doctor's diagnostic menu is one of the menus on the doctor's page. This diagnostic menu is used to view the record of symptoms that have been sent by the patient to the doctor. The doctor will see a list of Patients who consult with the doctor. Then the patient's message will be seen when the doctor chooses one of the names of his patients who have consulted. An example of a doctor diagnostic menu can be seen in Figure 9.
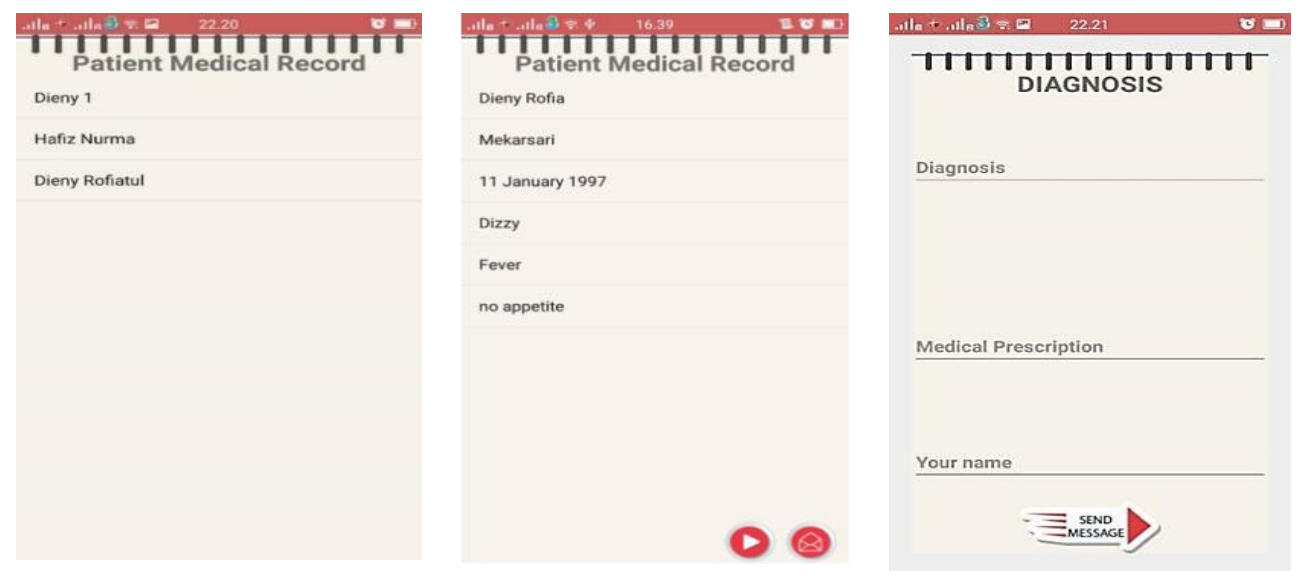

Figure 9. Diagnostic menu

\section{CONCLUSION}

This research has successfully implemented an embedded electronic stethoscope integrated with a mobile phone on the Android operating system. The electronic stethoscope works well in the $20 \mathrm{~Hz}-960 \mathrm{~Hz}$ frequency range according to the recommended heart or respiratory sound frequency. Volume control can be done through a variable resistor on board or an installed application. The electronic stethoscope can be connected with a loudspeaker or android mobile via a jack phone. The acquired sound is read by Steder

\footnotetext{
Implementation of electronic stethoscope for online remote monitoring with mobile... (Sugondo Hadiyoso)
} 
application installed on the Android phone. Steder can run well on the minimum Operating System: Ice Cream Sandwich. The application is integrated with Google Firebase as the cloud database server. All patient data will be stored in Firebase Real-Time Database, while the result of the sound recording will be stored in the Cloud Storage feature in Firebase. The patient can make consult with a doctor to get online real-time feedback. The size of Steder application in .apk format is $4.05 \mathrm{MB}$ and requires $42 \mathrm{MB}$ of RAM space when running.

In the future, if this system will be applied to real-world situations, several key aspects need to be considered including data security risks, storage media, device calibration, coverage areas, and error handling. In telehealth systems, these aspects need to be considered in detail. We will propose several approaches that might be considered to be applied to this system in the future. The security aspect can rely on existing encryption on the GSM-LTE network as an addition to common security (eg user accounts for authentication). Cloud computing in the context of data compression can be applied to the efficiency of storage devices. Another method is automatic data deletion at a certain period. Another aspect is the expansion of the internet service area, this is expected to be resolved quickly, because this is in line with government programs in the framework of providing communication services even in suburban areas. Finally, a remote monitoring system in health applications can be realized if all supporting infrastructure can be put together to build it. Another thing is continuous testing in real conditions until the system is established.

\section{REFERENCES}

[1] D. N. Reddy, vaijanatha v Yerigeri, M. Yadrami, and Harish Sanu, "Wireless Electronic Stethoscope Using Zigbee," Int. J. Eng. Sci. Res. Technol., vol. 4, no. 12, pp. 94-100, 2015.

[2] B. Malik, N. Eya, H. Migdadi, M. J. Ngala, and J. M. Noras, "Design and Development of an Electronic Stethoscope," in Internet Technologies and Applications (ITA), pp. 324-328, 2017.

[3] O. O. Fagbohun, "A versatile low cost Electronic Stethoscope design," IOSR J. Electron. Commun. Eng., vol. 10, no. 6, pp. 48-54, 2015.

[4] S. Leng, R. S. Tan, K. T. C. Chai, C. Wang, D. Ghista, and L. Zhong, “The electronic stethoscope," Biomed. Eng. Online, vol. 14, no. 1, pp. 1-37, 2015.

[5] I. D. G. H. Wisana, "Design Electronic Stethoscope for Cardiac Auscultation analyzed using Wavelet Decomposition,” Int. J. Comput. Networks Commun. Secur., vol. 1, no. 7, pp. 310-315, 2013.

[6] Y. Tang, G. Cao, H. Li, and K. Zhu, "The design of electronic heart sound stethoscope based on Bluetooth," in International Conference on Bioinformatics and Biomedical Engineering, pp. 1-4, 2010.

[7] A. S. Iskandar, A. S. Prihatmanto, and Y. Priyana, "Design and Implementation Electronic Stethoscope On Smart Chair for Monitoring Heart Rate and Stress Levels Driver," in International Conference on Interactive Digital Media (ICIDM), pp. 1-6, 2015.

[8] A. Kumari, P. Mohite, M. Zoye, and Bhushanmalkapurkar, “Advanced Electronic Stethoscope," Int. J. Sci. Res. Comput. Sci. Eng. Inf. Technol., vol. 3, no. 3, pp. 883-886, 2018.

[9] K. R. K. Patil, "Design of Wireless Electronic Stethoscope Based on Zigbee," Int. J. Distrib. Parallel Syst., vol. 3, no. 1, pp. 351-359, 2012.

[10] P. Parhana, M. Lakshmaiah, S. Allaudheen, S. Dastagiri, and M. V. Saritha, "Review on Internet of Things: Recent Applications and its Challenges," Int. J. Adv. Res. Electr. Electron. Instrum. Eng., vol. 6, no. 11, pp. 1-7, 2017.

[11] C. A. da Costa, C. F. Pasluosta, B. Eskofier, D. B. da Silva, and R. da Rosa Righi, "Internet of Health Things: Toward intelligent vital signs monitoring in hospital wards," Artif. Intell. Med., vol. 89, pp. 61-69, 2018.

[12] J. J. P. C. Rodrigues et al., "Enabling Technologies for the Internet of Health Things," in IEEE Access, vol. 6, pp. 13129-13141, 2018

[13] R. Basatneh, B. Najafi, and D. G. Armstrong, "Health Sensors, Smart Home Devices, and the Internet of Medical Things : An Opportunity for Dramatic Improvement in Care for the Lower Extremity Complications of Diabetes," J. Diabetes Sci. Technol., vol. 12, no. 3, pp. 577-586, 2018.

[14] E. Perrier, "Positive Disruption: Healthcare, Ageing \&Participation in the Age of Technology,"Australia: TheMcKell Institute, 2015.

[15] S. B. Baker, W. Xiang, and I. Atkinson, "Internet of Things for Smart Healthcare: Technologies, Challenges, and Opportunities," IEEE Access, vol. 5, pp. 26521-26544, 2017.

[16] Y. Jingjing et al., "Family health monitoring system based on the four sessions internet of things," TELKOMNIKATelecommunication Computing Electronics Control., vol. 13, no. 1, pp. 314-320, 2015.

[17] A. Rizal, V. Suryani, Jondri, and S. Hadiyoso, "Development of wireless patient's vital sign monitor using wireless LAN (IEEE.802.11.b/g) protocol," Intternational Journal Electrical Computer Engineering, vol. 4, no. 6, pp. 893-901, 2014.

[18] M. I. Sani, G. A. Mutiara, and R. S. D. W. Putra, "Fit-NES: Wearable bracelet for heart rate monitoring," Telkomnika (Telecommunication Comput. Electron. Control., vol. 17, no. 1, pp. 392-399, 2019.

[19] R. R. Adiputra, S. Hadiyoso, and Y. Sun Hariyani, "Internet of things: Low cost and wearable SpO2 device for health monitoring," Intternational Journal Electrical Computer Engineering., vol. 8, no. 2, pp. 939-945, 2018. 
[20] S. Misbahuddin, J. A. Zubairi, A. R. Alahdal, and M. A. Malik, "IoT-Based Ambulatory Vital Signs Data Transfer System,” J. Comput. Networks Commun., vol. 2018, 2018.

[21] M. Niswar, M. Nur, A. A. Ilham, and I. Mappangara, "A low cost wearable medical device for vital signs monitoring in low-resource settings," Intternational Journal Electrical Computer Engineering, vol. 9, no. 4, pp. 2321-2327, 2019.

[22] K. N. Swaroop, K. Chandu, R. Gorrepotu, and S. Deb, "A health monitoring system for vital signs using IoT," Internet of Things, vol. 5, pp. 116-129, 2019.

[23] M. Shu, M. Tang, M. Yang and N. Wei, "The Vital Signs Real-Time Monitoring System Based on Internet of Things," in 4th International Conference on Information Science and Control Engineering (ICISCE), pp. 747-751, 2017.

[24] A. GIORGIO, "the Last Generation of Electronic Stethoscopes: the Internet Tele-Stethoscope," Int. J. Bioinforma. Res., vol. 4, no. 1, pp. 249-253, 2012.

[25] I. Foche-perez et al., “An open real-time tele-stethoscopy system,” Biomed. Eng. Online, vol. 2012, pp. 1-17, 2012.

\section{BIOGRAPHIES OF AUTHORS}

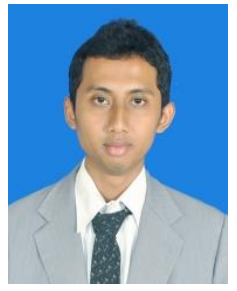

Sugondo Hadiyoso received the Master.in Electrical-Telecommunication Engineeringfrom Telkom University, Bandung, Indonesia in March 2012. He joined as a Lecturer in the department of Electronics and Communication Engineering of Telkom University, in 2010. where he is currently members of Biomedical Instrumentation Research Group in Telkom University. His research interests are wireless sensor network, embedded system, logic design on FPGA and biomedical engineering.

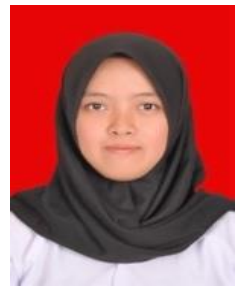

Dieny Rofiatul Mardiyah received an diploma degree in Telecommunications Engineering from Telkom University, Bandung, Indonesia in November 2018. Research interests are wireless sensor network and develop Android application. She is currently studying Bachelor degree at Telkom University, Bandung, Indonesia.

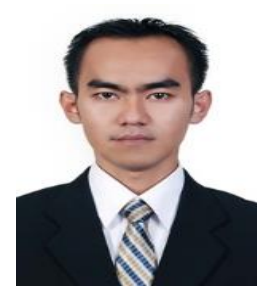

Dadan Nur Ramadan received the Bachelor of Education from Indonesian University of Education in 2006, then a Master in Telecommunication Engineering from Bandung Institute of Technology in 2014. He becomes a telecommunication technician in the PT. Indonesian telecommunications industry (INTI) from 2009 to 2013, and since 2014 currently active as Lecturer in the department of Electronics and Communication Engineering of Telkom University. Research interests on image processing, embedded system and Internet of Things.

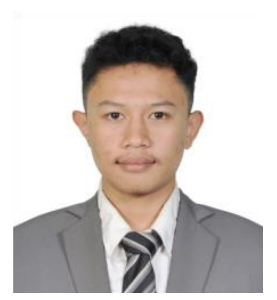

Asril Ibrahim received an diploma degree in Telecommunications Engineering from Telkom University, Bandung, Indonesia in November 2019. The work focus that he chose is the field of electrical technicians and networking. He has working experience as field engineer at PT. DatacommDiangraha. 